\title{
Activation of Naturally Occurring Lecithin:Cholesterol Acyltransferase Mutants by a Novel Activator Compound $\mathbf{s}$
}

\author{
Chiara Pavanello, ${ }^{1}$ Alice Ossoli, ${ }^{1}$ Marta Turri, Arianna Strazzella, Sara Simonelli, \\ Tommaso Laurenzi, Keita Kono, Keisuke Yamada, Naoki Kiyosawa, Ivano Eberini, \\ and Laura Calabresi
}

Centro E. Grossi Paoletti, Dipartimento di Scienze Farmacologiche e Biomolecolari (C.P., A.O., M.T., A.S., S.S., L.C.) and Dipartimento di Scienze Farmacologiche e Biomolecolari (T.L., I.E.), Università degli Studi di Milano, Milan, Italy; Specialty Medicine Research Laboratories I, Daiichi Sankyo Co., Ltd., Tokyo, Japan (K.K., N.K.); and Medical Affairs Planning Department, Daiichi Sankyo Co., Ltd., Tokyo, Japan (K.Y.)

Received June 9, 2020; accepted September 22, 2020

\begin{abstract}
Lecithin:cholesterol acyltransferase (LCAT) is a unique plasma enzyme able to esterify cholesterol, and it plays an important role in HDL maturation and promotion of reverse cholesterol transport. Familial LCAT deficiency (FLD; OMIM number 245900) is a rare recessive disease that results from loss-of-function mutations in the LCAT gene and has no cure. In this study, we assessed the in vitro efficacy of a novel small-molecule LCAT activator. Cholesterol esterification rate (CER) and LCAT activity were tested in plasma from six controls and five FLD homozygous carriers of various LCAT mutations at different doses of the compound $(0.1,1$, and $10 \mu \mathrm{g} / \mathrm{ml})$. In control plasma, the compound significantly increased both CER $(P<0.001)$ and LCAT activity $(P=0.007)$ in a dose-dependent manner. Both CER and LCAT activity increased by 4 - to 5-fold, reaching maximum activation at the dose of $1 \mu \mathrm{g} / \mathrm{ml}$. Interestingly, Daiichi Sankyo
\end{abstract}

compound produced an increase in CER in two of the five tested LCAT mutants (Leu372--Arg and Val309--Met), while LCAT activity increased in three LCAT mutants (Arg147--Trp, Thr274--Ile and Leu372--Arg); mutant Pro254--Ser was not activated at any of the tested doses. The present findings form the basis for personalized therapeutic interventions in FLD carriers and support the potential LCAT activation in secondary LCAT defects.

\section{SIGNIFICANCE STATEMENT}

We characterized the pharmacology of a novel small-molecule LCAT activator in vitro on a subset of naturally occurring LCAT mutants. Our findings form the basis for personalized therapeutic interventions for familial LCAT deficiency carriers, who can face severe complications and for whom no cure exists.

\section{Introduction}

Familial lecithin:cholesterol acyltransferase (LCAT) deficiency (FLD; OMIM \#245900) is a rare autosomal recessive disorder that results from loss-of-function mutations in the $L C A T$ gene. In homozygous and compound heterozygous carriers, the lack of LCAT catalytic activity is complete, thereby affecting the ability of the enzyme to esterify free cholesterol on all plasma lipoproteins (Calabresi et al., 2012). As a consequence, cholesteryl esters are virtually absent in plasma. Clinical manifestations of FLD include corneal opacity, hemolytic anemia, proteinuria, and renal disease (Calabresi et al., 2012). Of note, glomerulosclerosis is the major cause of morbidity and mortality in FLD cases and may ultimately lead to renal failure in early life (from 30 years of

\footnotetext{
This work was supported in part by a grant from Daiichi Sankyo (to L.C.). K.K., K. Y., and N. K. are employees of Daiichi Sankyo.

${ }^{1}$ C.P. and A.O. contributed equally to this work.

https://doi.org/10.1124/jpet.120.000159.

S This article has supplemental material available at jpet.aspetjournals.org.
}

age onward) (Santamarina-Fojo et al., 2001). Renal transplantation represents an option in severe cases with end-stage renal disease, but the disease can rapidly reoccur in the transplanted kidney within only a few years (Strom et al., 2011). Hence, there is no cure for FLD, and the present treatment consists of currently available therapies mainly to delay the evolution of chronic nephropathy. Enzyme replacement therapy with recombinant human LCAT (rhLCAT) is the most clinically advanced therapy for FLD (Shamburek et al., 2016a,b), but it is expensive, requires intravenous infusion, and can cause immunogenicity in treated patients, which is a relevant issue in chronic treatments. Small-molecule activators able to partially restore enzyme activity in LCATdeficient patients represent a possible alternative to rhLCAT. Compound $\mathrm{A}$ is a previously described small molecule that proved to activate LCAT in vitro (Chen et al., 2012; Freeman et al., 2017) and to increase plasma cholesterol ester and HDL cholesterol levels in mice and hamsters (Chen et al., 2012). A novel class of reversible piperidinylpyrazolopyridine and piperidinylimidazopyridine activators were reported to activate

ABBREVIATIONS: apoA-I, apolipoprotein A-I; apoB, apolipoprotein B; apoB-D, apolipoprotein B-depleted; CER, cholesterol esterification rate; DS, Daiichi Sankyo; FLD, familial LCAT deficiency; HDL, high-density lipoprotein; LCAT, lecithin:cholesterol acyltransferase; LDL, low-density lipoprotein; MD, molecular dynamics; rhLCAT, recombinant human LCAT; VLDL, very low-density lipoprotein. 


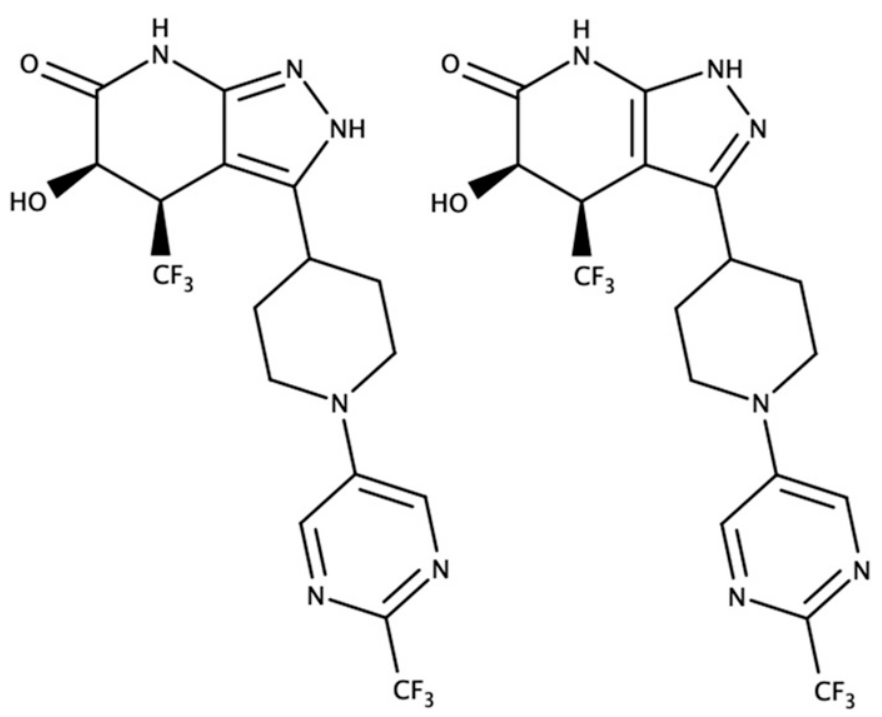

Fig. 1. Chemical structure of DS compound.

human LCAT (patent number US9796709B2) and to increase HDL cholesterol when administered to cynomolgus monkeys (WO2015/087996). Activators from this series have been shown to bind membrane binding domain in LCAT without altering HDL binding capacity (Manthei et al., 2018). The purpose of the current study was to characterize the pharmacology of a novel LCAT activator (DS compound, Daiichi Sankyo) in vitro on a subset of naturally occurring LCAT mutants.

\section{Materials and Methods}

The raw data that support the findings of this study are available from the corresponding author upon reasonable request.

Subjects. Five homozygous carriers (two males and three females; age $43 \pm 6$ years) of different $L C A T$ mutations, belonging to the Italian cohort (Calabresi et al., 2005, 2012), and six healthy donors (control subjects; three males and three females; age $41 \pm 17$ years) were asked to participate in the study. All the subjects were fully informed of the modalities and endpoints of the study and signed an informed consent. The study was approved by the Ethical Committee of the University of Milan (approval number 43/17) and conformed to the guidelines set out in the Declaration of Helsinki.

Activator. DS compound (international publication number: WO2015/087994) (Fig. 1) was synthesized and stored at $-20^{\circ} \mathrm{C}$ and protected from light. The compound was solubilized in DMSO prior to use.

Biochemical Analyses. Blood samples were collected in EDTA tubes after an overnight fast, and plasma was separated by low-speed centrifugation at $4^{\circ} \mathrm{C}$. Plasma total cholesterol, HDL cholesterol, triglyceride, and apolipoprotein levels were determined with certified methods using a Roche Integra c311 AutoAnalyzer (Roche Diagnostics). LDL cholesterol was calculated using Friedewald's formula. When triglycerides were $>400 \mathrm{mg} / \mathrm{dl}$, LDL cholesterol was assessed using direct measurement. Unesterified cholesterol was determined by using a previously described enzymatic technique (Ossoli et al., 2019). Plasma LCAT concentration was measured by a specific competitive enzyme-linked immunoassay (Murakami et al., 1995). Plasma cholesterol esterification rate (CER) and LCAT activity, which reflect the ability of endogenous LCAT to esterify cholesterol within endogenous lipoproteins and exogenous HDL, respectively, were assessed by using previously described methods (Calabresi et al., 2005). LCAT activity and CER in plasma of subjects with FLD and controls were assessed in the presence or absence of the DS compound at three different doses $(0.1,1$, and $10 \mu \mathrm{g} / \mathrm{ml})$. The apolipoprotein B-depleted (apoB-D) plasma was obtained from the whole plasma by precipitating the apoB-containing lipoproteins with a polyethylene glycol solution, as already described (Arnaboldi et al., 2020). Shortly, whole plasma was incubated for 20 minutes with a $20 \%$ polyethylene glycol 6000 solution. After sample centrifugation at $10,000 \mathrm{rpm}$, the supernatant was collected and stored at $4^{\circ} \mathrm{C}$.

LCAT activity on apoB-D plasma from subjects with FLD and controls was tested only at the dose of $10 \mu \mathrm{g} / \mathrm{ml}$ of activator, which was confirmed to be the enzyme activating dose in LCAT-deficient plasma. All substrates were assayed in triplicate, with the average used for subsequent analysis.

Molecular Modeling and Simulation Analyses. The initial LCAT::DS compound complex was generated using the crystallographic structure published by Manthei et al. (2018) (Protein Data Bank ID: 6MVD), in which LCAT is cocrystalized with a drug analog of DS compound. DS compound was then superposed on the crystalized ligand, and the complex was refined with an all-atom minimization to convergence. DS compound binding site was also validated by probing the entire LCAT surface with molecular docking (see Supplemental Material). Molecular dynamics (MD) simulations were run to assess the impact of DS compound on LCAT dynamic and structural features, comparing two 500-nanosecond-long simulations run in the presence or absence of the ligand of interest. LCAT modeling, simulations, and analyses were performed using the Schrödinger Small Molecule Drug Discovery Suite 2020-2 with the OPLS3e force field (2020; Schrödinger, LLC, New York, NY).

Statistical Analyses. Data are presented as means \pm S.D., unless otherwise stated. Changes versus baseline are presented as absolute differences in activity. Comparisons between different activator doses in control subjects were performed by one-way ANOVA for repeated measures followed by Bonferroni correction. Tests were two-sided, and $P$ values $<0.05$ were considered statistically significant. Statistical analysis was performed using SPSS version 24.0 software (SPSS Inc., Chicago).

\section{Results}

Plasma lipid levels in the examined subjects are summarized in Table 1. FLD carriers enrolled in the present study displayed the peculiar lipid profile described in homozygous FLD carriers (Calabresi et al., 2012). HDL cholesterol, apolipoprotein A-I, and apolipoprotein B levels were dramatically reduced in all carriers, whereas triglyceride levels were increased. Plasma unesterified cholesterol levels were increased and, on average, were three times higher in carriers compared with controls. As expected, CER and LCAT activity were null in all carriers. LCAT mass was reduced but measurable in all FLD carriers (Table 1).

DS Compound Increases LCAT Activity in Plasma of Control Subjects. Even at the lowest dose, DS compound significantly increased both CER and LCAT activity in all tested control plasma samples. Indeed, the mean basal CER of $44.1 \pm 16.3 \mathrm{nmol} / \mathrm{ml}$ per hour doubled to $86.1 \pm 24.9 \mathrm{nmol} / \mathrm{ml}$ per hour $(P<0.001)$ at the dose of $0.1 \mu \mathrm{g} / \mathrm{ml}$. The activator leads to a 3-fold increase in CER at the dose of $1 \mu \mathrm{g} / \mathrm{ml}$, up to $121.5 \pm 43.0 \mathrm{nmol} / \mathrm{ml}$ per hour $(+166.2 \% \pm 77.0 \%, P<0.001)$. No further activation was observed at the dose of $10 \mu \mathrm{g} / \mathrm{ml}$, with CER remaining stable at $122.1 \pm 42.8 \mathrm{nmol} / \mathrm{ml}$ per hour $(+199.8 \% \pm 67.0 \% \mathrm{nmol} / \mathrm{ml}$ per hour, $P<0.001)($ Fig. $2 A)$. In the same way, the mean basal LCAT activity in control plasma increased from $35.6 \pm 16.3$ to $92.5 \pm 50.6 \mathrm{nmol} / \mathrm{ml}$ per hour $(+159.2 \% \pm 70.9 \%, P=0.035)$ at the dose of $0.1 \mu \mathrm{g} / \mathrm{ml}$ and to $140.1 \pm 72.3 \mathrm{nmol} / \mathrm{ml}$ per hour $(+295.2 \% \pm 106.4 \%, P=0.007)$ 
TABLE 1

Characteristics of controls and FLD carriers

Values are expressed as means \pm S.D.

\begin{tabular}{|c|c|c|c|c|c|c|}
\hline Laboratory Parameters & Controls $(n=6)$ & Arg147--Trp & Thr274--Ile & Leu372--Arg & Pro254--Ser & Val309--Met \\
\hline Total cholesterol (mg/dl) & $160.8 \pm 33.2$ & 74 & 216 & 62 & 243 & 140 \\
\hline Unesterified cholesterol (mg/dl) & $46.2 \pm 9.5$ & 74 & 193 & 62 & 191 & 124 \\
\hline LDL cholesterol (mg/dl) & $80.0 \pm 23.0$ & 44 & 156 & 26 & 104 & 56 \\
\hline HDL cholesterol (mg/dl) & $65.2 \pm 14.6$ & 13 & 4 & 16 & 7 & 7 \\
\hline Triglycerides (mg/dl) & $77.0 \pm 24.9$ & 85 & 278 & 98 & 658 & 337 \\
\hline Apolipoprotein A-I (mg/dl) & NA & 37 & 51 & 20 & 38 & 30 \\
\hline Apolipoprotein B (mg/dl) & NA & 47 & 58 & 25 & 108 & 56 \\
\hline CER (nmol/ml per hour) & $44.1 \pm 16.3$ & 0 & 0 & 0 & 0 & 0 \\
\hline LCAT activity (nmol/ml per hour) & $35.6 \pm 16.3$ & 0 & 0 & 0 & 0 & 0 \\
\hline LCAT concentration $(\mu \mathrm{g} / \mathrm{ml})$ & $5.4 \pm 0.8$ & 1.7 & 0.8 & 2.7 & 1.0 & 0.9 \\
\hline
\end{tabular}

NA, not available.

at the dose of $1 \mu \mathrm{g} / \mathrm{ml}$. Again, no further activation was observed at the dose of $10 \mu \mathrm{g} / \mathrm{ml}(+245.7 \% \pm 110.7 \%, P=0.012)$ (Fig. 2B). Specific LCAT activity, calculated dividing LCAT activity by plasma LCAT concentration, increased from 5.4 to $18.6 \mathrm{mmol} / \mu \mathrm{g}$ per hour at the maximum tested dose. These results clearly show that the DS compound is a potent activator of human LCAT in a dose-dependent manner, reaching a plateau at the dose of $1 \mu \mathrm{g} / \mathrm{ml}$ (Fig. 2).

In addition, to verify the effect of DS activator on endogenous HDL, we tested apoB-D plasma as substrate instead of synthetic HDL. DS compound at the tested dose of $10 \mu \mathrm{g} / \mathrm{ml}$ increased LCAT activity from $31.5 \pm 3.5$ to $55.4 \pm 1.3 \mathrm{nmol} / \mathrm{ml}$ per hour (+76.8\%, Supplemental Fig. 1).

DS Compound Stabilizes the LCAT Lid Loop. The analysis of protein-ligand interactions during the MD simulation highlighted key residues involved in the molecular recognition mechanism of DS compound by LCAT. In particular, persistent hydrogen bonds ( $>90 \%$ of the simulated time) form between DS compound and Asp63, Asn78, and Try51, including also a $\pi-\pi$ stacking with the DS condensed rings, whereas Leu68 and Cys74 contribute with hydrophobic interactions. The dynamic behavior of residues of the apo and the complexed (with DS compound) forms of LCAT show that the mobility of DS compound binding pocket remains unaltered, whereas residues $45-47$ of the membrane binding domain gain increased mobility; moreover, the lid loop, responsible for regulating the accessibility of substrates to LCAT binding site, is much more stable in an open conformation when DS compound is bound to LCAT (Fig. 3; Supplemental Figs. 2 and 3).

DS Compound Increases LCAT Activity for Some Naturally Occurring LCAT Mutants. The ability of DS compound to activate LCAT mutants, all showing complete enzymatic defect, was tested in plasma from five subjects with FLD carrying different LCAT mutations (Fig. 4; Table 2). Mutant Arg147--Trp was activated by DS compound at the dose of $10 \mu \mathrm{g} / \mathrm{ml}$, with LCAT activity increasing from 0 to 26.6 $\mathrm{nmol} / \mathrm{ml}$ per hour; on the contrary, CER remained null at all tested doses. Specific LCAT activity of the Arg147-Trp mutant reached a value of $14.7 \mathrm{mmol} / \mu \mathrm{g}$ per hour, which was similar to that reached by wild-type LCAT. Mutant Thr274--Ile was also activated by the DS compound; at the dose of $0.1 \mu \mathrm{g} / \mathrm{ml}$, LCAT activity increased to $31.2 \mathrm{nmol} / \mathrm{ml}$ per hour, and the activation was maintained at the higher doses (27.5 and $34.4 \mathrm{nmol} / \mathrm{ml}$ per hour, respectively). Specific LCAT activity of the Thr274--Ile mutant reached a value of 47.1 $\mathrm{mmol} / \mu \mathrm{g}$ per hour at the highest dose of the activator, thus showing that activation was independent of LCAT mass. Also, for this mutant, CER remained null at all tested doses. Trying to clarify these apparently conflicting results, we tested the ability of the DS compound to activate the two LCAT mutants measuring LCAT activity on endogenous HDL (apoB-D

B

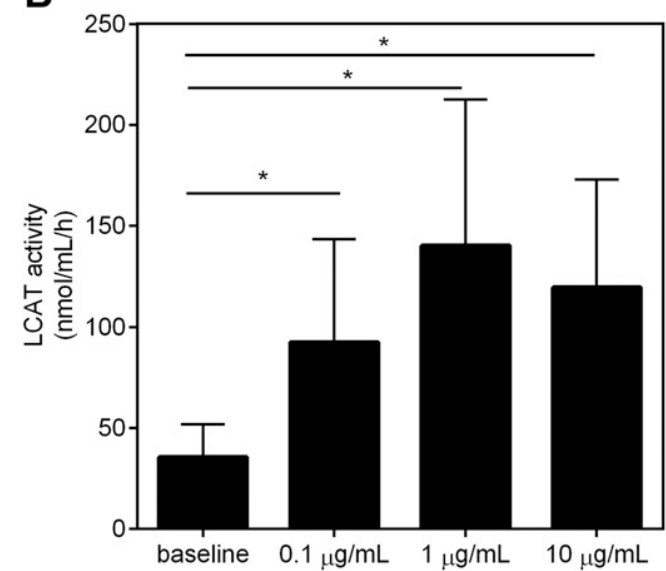

Fig. 2. Dose-response for CER (A) and LCAT activity (B) in control plasma. CER and LCAT activity were measured in plasma from $n=6$ control subjects before (baseline) and after incubation with DS compound at different doses. The differences in the mean values among the treatment doses were analyzed by one-way ANOVA for repeated measures, followed by Bonferroni correction for multiple comparisons. Data are expressed as means \pm S.D. $* * P<0.01 ; * P<0.05$ 


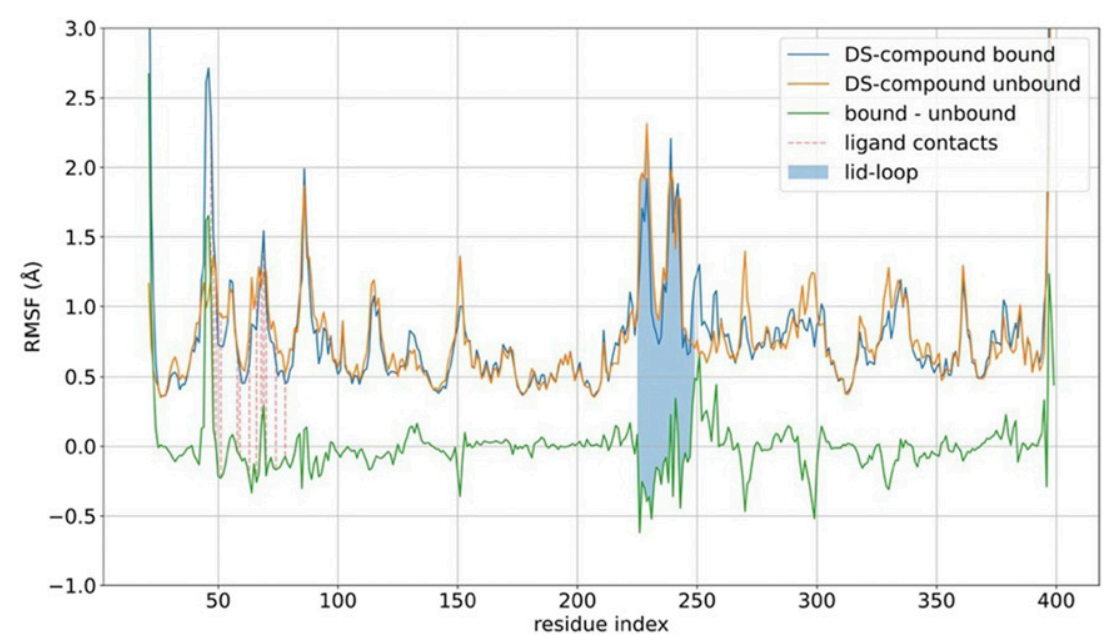

Fig. 3. Comparison between LCAT apo and DS compound bound forms. The root mean squared fluctuation (RMSF) values of LCAT residues during MD simulations are reported. RMSF is a measure of a residue's overall mobility. Residues interacting with DS compound and the lid loop are highlighted. plasma). As shown in Supplemental Fig. 1, no activation was observed, thus suggesting that the DS compound is able to activate the mutant enzyme, leading to cholesterol esterification on exogenous (synthetic) HDL, which has no effect on endogenous lipoproteins (both apoB- and apoA-I-containing). Mutant Leu372--Arg was activated by the DS compound at the highest tested dose, leading to cholesterol esterification on all tested substrates (endogenous and exogenous lipoproteins). Specific LCAT activity of the Leu372--Arg mutant reached a value of $5.7 \mathrm{mmol} / \mu \mathrm{g}$ per hour at the highest dose of the activator. In addition, when apoB-D plasma was tested, LCAT activity increased to $9.2 \mathrm{nmol} / \mathrm{ml}$ per hour (Supplemental Fig. 4). The Val309--Met mutant was also activated at the highest tested dose of the DS compound. Although CER increased up to $20.3 \mathrm{nmol} / \mathrm{ml}$ per hour, LCAT activity did not change (Fig. 4; Table 2 ), thus suggesting that only the $\beta$-activity of LCAT is increased. Unfortunately, no more sample was available from this subject to test the apoB-D plasma. Mutant Pro254--Ser was not activated by DS compound at any of the tested doses, despite a plasma concentration similar to that of other tested mutants (Fig. 4; Table 2).

\section{Discussion}

FLD is a rare hereditary disease with high morbidity and no treatment. Phenotypic manifestations could be highly heterogeneous depending on the underlying mutation and metabolic and environmental factors. The use of rhLCAT has been previously investigated as a potential therapy, and it has been demonstrated to be safe and well tolerated (Shamburek et al., 2016a); however, a recombinant protein is expensive and requires intravenous infusions, which makes chronic use demanding. In this context, small-molecule LCAT activators, which are able to activate LCAT mutants causing FLD, could represent a better treatment option,. The therapeutic use of small-molecules offers several advantages to patients, including an oral administration route and no need for inpatient treatment, leading to an improved quality of life and lower social costs. Previously tested compound A, the first developed LCAT activator, has already been shown to increase LCAT activity and raise HDL cholesterol levels in the plasma of wildtype mice and hamsters (Chen et al., 2012). When tested in vitro using plasma of FLD carriers, it was able to activate one out of four naturally occurring LCAT mutants.

Here, we have tested a novel compound, taking advantage of the availability of plasma samples from a large cohort of carriers of LCAT mutations (Calabresi et al., 2005, 2012). To better characterize the effect of the compound on the esterification process, we measured both CER and LCAT activity. Whereas the first reflects the ability of LCAT to esterify cholesterol on endogenous lipoproteins (HDL and apoBcontaining particles), the latter measures the esterification on synthetic HDL (Calabresi et al., 2005). DS compound enhanced activity of wild-type LCAT up to 5.6-fold when
A

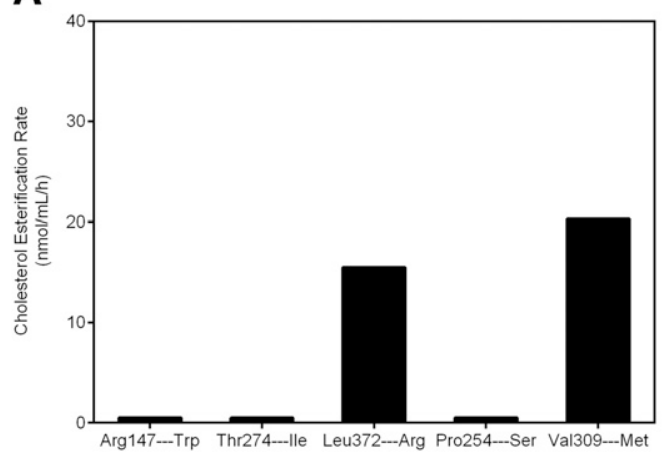

B

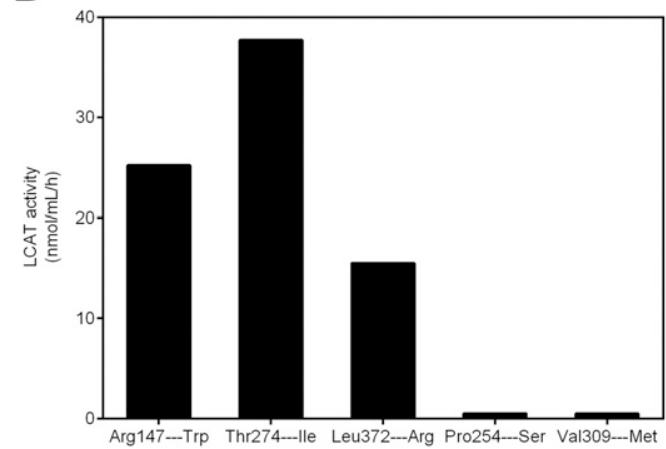

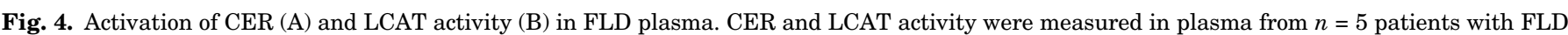

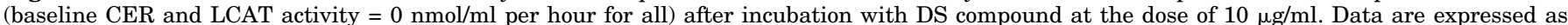
absolute increases. 
TABLE 2

CER and LCAT activity in FLD plasma before and after incubation with DS compound

\begin{tabular}{|c|c|c|c|c|c|c|c|c|}
\hline \multirow[b]{3}{*}{ Mutation } & \multicolumn{4}{|c|}{ CER (nmol/ml Per Hour) } & \multicolumn{4}{|c|}{ LCAT Activity (nmol/ml Per Hour) } \\
\hline & \multirow{2}{*}{ Baseline } & \multicolumn{3}{|c|}{ DS Compound } & \multirow{2}{*}{ Baseline } & \multicolumn{3}{|c|}{ DS Compound } \\
\hline & & $0.1 \mu \mathrm{g} / \mathrm{ml}$ & $1 \mu \mathrm{g} / \mathrm{ml}$ & $10 \mu \mathrm{g} / \mathrm{ml}$ & & $0.1 \mu \mathrm{g} / \mathrm{ml}$ & $1 \mu \mathrm{g} / \mathrm{ml}$ & $10 \mu \mathrm{g} / \mathrm{ml}$ \\
\hline Arg147Trp & 0.0 & 0.0 & 0.0 & 0.0 & 0.0 & 0.0 & 0.0 & 25.2 \\
\hline Thr274Ile & 0.0 & 0.0 & 0.0 & 0.0 & 0.0 & 25.8 & 27.6 & 37.7 \\
\hline Leu372Arg & 0.0 & 0.0 & 0.0 & 24.1 & 0.0 & 0.0 & 15.5 & 15.5 \\
\hline Pro254Ser & 0.0 & 0.0 & 0.0 & 0.0 & 0.0 & 0.0 & 0.0 & 0.0 \\
\hline Val309Met & 0.0 & 21.7 & 10.6 & 20.3 & 0.0 & 0.0 & 0.0 & 0.0 \\
\hline
\end{tabular}

measured as CER and up to 4.2-fold in LCAT activity, thus demonstrating efficacy in augmenting LCAT ability to esterify cholesterol in all substrates, even at the dose of $1 \mu \mathrm{g} / \mathrm{ml}$. According to our molecular modeling data, DS compound activity is related to its ability to stabilize the LCAT lid loop in an open conformation and to enhance the hydrophobicity of the LCAT membrane binding domain, thus facilitating its interaction with lipids. These data allow us to hypothesize a potential increase of the LCAT $V_{\max }$ without significantly affecting the $\mathrm{K}_{\mathrm{m}}$, as described also for compound $\mathrm{A}$ (Freeman et al., 2017).

The efficacy of the compound to activate naturally occurring LCAT mutants was then tested, selecting FLD homozygous carriers belonging to the large Italian cohort. DS compound produced an increase in CER in two of the five tested FLD mutations, whereas LCAT activity increased in three FLD mutations. Only one of the tested mutants, the Pro254--Ser, which introduces a hydrophilic residue within a pack of hydrophobic residues close to the lid loop region, likely disrupting LCAT fold, was not activated by DS compound in any experimental conditions, thus likely excluding carriers of this variant from benefit from this activator. DS compound activated similarly Arg147--Trp and Thr274--Ile mutants by increasing LCAT activity and not CER, thus suggesting that the DS compound activates the ability of the mutant LCAT to esterify cholesterol only on synthetic HDL, having no effect on endogenous lipoproteins. This was confirmed by the absence of esterification activity on apoB-D plasma (endogenous HDL) of the same carriers. Circulating HDL particles are largely heterogenous, containing different phospholipids and proteins. Although synthetic HDL resembles nascent HDL particles, they are not representative of the complex diversity of circulating HDL and contain only apoA-I and a single phospholipid species. Mutation Arg147--Trp is in a region involved in phospholipid binding in active site. Thr274--Ile, despite being far from the LCAT active site, has instead been predicted to associate with a steric bulk, which causes a local rearrangement decreasing the lipoprotein binding area (Sensi et al., 2014). Among tested LCAT mutants, Leu372--Arg, which falls in a region likely involved in apoA-I recognition (Manthei et al., 2018), was the only mutant to be activated in all tested conditions (CER, LCAT activity on synthetic HDL, and LCAT activity on apoB-D plasma). DS compound at the dose of $1 \mu \mathrm{g} / \mathrm{ml}$ was already able to boost LCAT activity on a simple form of HDL, as synthetic HDL, whereas higher doses were required to activate the more complex plasma lipoproteins (apoB-D plasma). The Val309--Met mutant, a mutation far from the active site and from lipoprotein binding site, was activated only on endogenous substrates, already at the lowest dose of the DS compound, whereas no effect of the activator was observed on synthetic HDL. This suggest that only the activity on apoB-containing lipoproteins (VLDL/LDL), but not on HDL, is increased. Whether this may be beneficial or not needs to be further addressed by in vivo studies. Indeed, evidence from LCAT-deficient carriers supports the observation that increasing cholesteryl esters in VLDL/LDL is not beneficial, since it would possibly increase their atherogenic properties (Oldoni et al., 2018). On the other hand, it will remove unesterified cholesterol from the circulation and possibly reverse renal disease in subjects with FLD by reducing the formation of a nephrotoxic lipoprotein called lipoprotein X. Lipoprotein X is enriched in free cholesterol and was found to accumulate in the kidney, where it induces the peculiar glomerular damage (O et al., 1997; Ossoli et al., 2016).

In conclusion, the DS compound is a novel LCAT activator able to increase in a dose-dependent manner LCAT ability to esterify cholesterol within all substrates. The use of an LCAT activator could be beneficial in conditions characterized by secondary LCAT defects, such as acute coronary syndrome (Ossoli et al., 2019), and chronic kidney disease (Calabresi et al., 2015; Baragetti et al., 2020). Interestingly, the compound can also activate some naturally occurring LCAT mutants, rendering them able to esterify cholesterol in some or all lipoprotein substrates. Animal studies are required to prove that the activation of LCAT mutants by the DS compound will lead to a reduction in circulating unesterified cholesterol, which is the goal of therapy in patients with FLD. The present results thus form the basis for a personalized therapeutic intervention in FLD carriers, who can face severe clinical complications and for whom no cure exists.

\section{Acknowledgments}

The authors thank Drs. Takahiro Shibayama, Toshio Kaneko, and Atsuhiro Sugidachi for their contributions to the LCAT activator project in Daiichi Sankyo and support for the current research.

\section{Authorship Contributions}

Participated in research design: Pavanello, Ossoli, Kono, Yamada, Kiyosawa, Eberini, Calabresi.

Conducted experiments: Turri, Strazzella, Simonelli, Laurenzi.

Performed data analysis: Pavanello, Simonelli.

Wrote or contributed to the writing of the manuscript: Pavanello, Ossoli, Turri, Kiyosawa, Eberini, Calabresi.

\section{References}

Arnaboldi L, Ossoli A, Giorgio E, Pisciotta L, Lucchi T, Grigore L, Pavanello C, Granata A, Pasta A, Arosio B, et al. (2020) LIPA gene mutations affect the composition of lipoproteins: enrichment in ACAT-derived cholesteryl esters. Atherosclerosis 297:8-15.

Baragetti A, Ossoli A, Strazzella A, Simonelli S, Baragetti I, Grigore L, Pellegatta F, Catapano AL, Norata GD, and Calabresi L (2020) Low plasma lecithin: cholesterol acyltransferase (LCAT) concentration predicts chronic kidney disease. J Clin Med 9:2289. 
Calabresi L, Pisciotta L, Costantin A, Frigerio I, Eberini I, Alessandrini P, Arca M, Bon GB, Boscutti G, Busnach G, et al. (2005) The molecular basis of lecithin: cholesterol acyltransferase deficiency syndromes: a comprehensive study of molecular and biochemical findings in 13 unrelated Italian families. Arterioscler Thromb Vasc Biol 25:1972-1978.

Calabresi L, Simonelli S, Conca P, Busnach G, Cabibbe M, Gesualdo L, Gigante M, Penco S, Veglia F, and Franceschini G (2015) Acquired lecithin:cholesterol acyltransferase deficiency as a major factor in lowering plasma HDL levels in chronic kidney disease. J Intern Med 277:552-561.

Calabresi L, Simonelli S, Gomaraschi M, and Franceschini G (2012) Genetic lecithin cholesterol acyltransferase deficiency and cardiovascular disease. Atherosclerosis 222:299-306.

Chen Z, Wang SP, Krsmanovic ML, Castro-Perez J, Gagen K, Mendoza V, Rosa R, Shah V, He T, Stout SJ, et al. (2012) Small molecule activation of lecithin cholesterol acyltransferase modulates lipoprotein metabolism in mice and hamsters. Metabolism 61:470-481.

Freeman LA, Demosky SJ Jr, Konaklieva M, Kuskovsky R, Aponte A, Ossoli AF Gordon SM, Koby RF, Manthei KA, Shen M, et al. (2017) Lecithin:cholesterol acyltransferase activation by sulfhydryl-reactive small molecules: role of cysteine31. J Pharmacol Exp Ther 362:306-318.

Manthei KA, Yang SM, Baljinnyam B, Chang L, Glukhova A, Yuan W, Freeman LA Maloney DJ, Schwendeman A, Remaley AT, et al. (2018) Molecular basis for activation of lecithin:cholesterol acyltransferase by a compound that increases HDL cholesterol. eLife 7:e41604.

Murakami T, Michelagnoli S, Longhi R, Gianfranceschi G, Pazzucconi F, Calabresi L Sirtori CR, and Franceschini G (1995) Triglycerides are major determinants of cholesterol esterification/transfer and HDL remodeling in human plasma. Arte rioscler Thromb Vasc Biol 15:1819-1828.

O K, Ly M, Fang DZ, Frohlich J, and Choy PC (1997) Effect of lipoprotein-X on lipid metabolism in rat kidney. Mol Cell Biochem 173:17-24

Oldoni F, Baldassarre D, Castelnuovo S, Ossoli A, Amato M, van Capelleveen J, Hovingh GK, De Groot E, Bochem A, Simonelli S, et al. (2018) Complete and partial lecithin:cholesterol acyltransferase deficiency is differentially associated with atherosclerosis. Circulation 138:1000-1007.

Ossoli A, Neufeld EB, Thacker SG, Vaisman B, Pryor M, Freeman LA, Brantner CA Baranova I, Francone NO, Demosky SJ Jr, et al. (2016) Lipoprotein X causes renal disease in LCAT deficiency. PLoS One 11:e150083.

Ossoli A, Simonelli S, Varrenti M, Morici N, Oliva F, Stucchi M, Gomaraschi M, Strazzella A Arnaboldi L, Thomas M.J, et al. (2019) Recombinant LCAT (lecithin cholesterol acyltransferase) rescues defective HDL (high-density lipoprotein)-mediated endothelial protection in acute coronary syndrome. Arterioscler Thromb Vasc Biol 39:915-924.

Santamarina-Fojo S, Hoeg JM, Assmann G, and Brewer HBJ (2001) Lecithin cholesterol acyltransferase deficiency and fish eye disease, in The Metabolic and $M o$ lecular Bases of Inherited Diseases (Scriver CR, Beaudet AL, Sly WS, and Valle D eds) pp 2817-2833, McGraw-Hill, New York.

Sensi C, Simonelli S, Zanotti I, Tedeschi G, Lusardi G, Franceschini G, Calabresi L, and Eberini I (2014) Distant homology modeling of LCAT and its validation through in silico targeting and in vitro and in vivo assays. PLoS One 9:e95044.

Shamburek RD, Bakker-Arkema R, Auerbach BJ, Krause BR, Homan R, Amar MJ, Freeman LA, and Remaley AT (2016a) Familial lecithin:cholesterol acyltransferase deficiency: first-in-human treatment with enzyme replacement. J Clin Lipidol 10: 356-367.

Shamburek RD, Bakker-Arkema R, Shamburek AM, Freeman LA, Amar MJ, Auerbach B, Krause BR, Homan R, Adelman SJ, Collins HL, et al. (2016b) Safety and tolerability of ACP-501, a recombinant human lecithin:cholesterol acyltransferase, in a phase 1 single-dose escalation study. Circ Res 118:73-82.

Strøm EH, Sund S, Reier-Nilsen M, Dørje C, and Leren TP (2011) Lecithin: cholesterol acyltransferase (LCAT) deficiency: renal lesions with early graft recurrence. Ultrastruct Pathol 35:139-145.

Address correspondence to: Dr. Laura Calabresi, Center Grossi Paoletti, Department of Pharmacological Sciences, Università degli Studi di Milano, Via Balzaretti 9, 20133 Milano, Italy. E-mail: laura.calabresi@unimi.it 